\title{
ON THE GROUP RING OF A FREE PRODUCT WITH AMALGAMATION
}

\author{
by CAMILLA R. JORDAN
}

(Received 2 April, 1979)

1. Introduction. Let $G=A *_{H} B$ be the free product of the groups $A$ and $B$ amalgamating the proper subgroup $H$ and let $R$ be a ring with 1 . If $H$ is finite and $G$ is not finitely generated we show that any non-zero ideal $I$ of $R(G)$ intersects non-trivially with the group ring $R(M)$, where $M=M(I)$ is a subgroup of $G$ which is a free product amalgamating a finite normal subgroup. This result compares with A. I. Lichtman's results in [6] but is not a direct generalisation of these.

We then apply this theorem together with results in [4] and [1] to obtain the following theorems on $J R(G)$, the Jacobson radical of $R(G)$, and on $Z R(G)$, the right singular ideal of $R(G)$. We denote by $N R\left(\triangle^{+}(G)\right)$ the nilpotent radical of $R\left(\triangle^{+}(G)\right.$ ).

TheORem. Let $G=A *_{H} B$, where $H$ is a finite group, and let $R$ be a right noetherian ring with 1 . If $G$ is not finitely generated then

(i) $R(G)$ is semiprimitive if and only if $R(G)$ is semiprime,

(ii) if $R$ is a field, $J R(G)=N R\left(\Delta^{+}(G)\right) R(G)$.

Theorem. Let $G=A *_{H} B$, where $H$ is a finite group, and let $K$ be a field. If $G$ is not finitely generated then $Z K(G)=N K(G)$.

Our notation will be that usually employed. In particular, $A *_{H} B$ will denote the free product of groups $A, B$ amalgamating the subgroup $H ;|A: H|$ will denote the number of cosets of $H$ in $A$. If we choose right transversals $S$, T, respectively, for $A, B$ modulo $H$ then every element $g \in G=A *_{H} B$ can be written uniquely in the form

$$
g=h a_{1} b_{1} a_{2} b_{2} \ldots a_{n} b_{n}
$$

where $h \in H, a_{i} \in S, b_{j} \in T, a_{i} \neq 1$ if $i \neq 1$ and $b_{i} \neq 1$ if $j \neq n$. This is called the normal form of $\mathrm{g}\left[7, \mathrm{p}\right.$. 205]. If $a_{1} \neq 1 \neq b_{n}$ we say that $\mathrm{g}$ has $A B$ form. We define similarly $A A, B A$ and $B B$ form for elements of $G$. If $b_{n} \neq 1$ we say $g$ has $-B$ form. We define $-A, B-$, and $A-$ form for elements of $G$ in the same way.

Part of this work appears in the author's Ph.D. thesis presented to the University of Leeds. The author wishes to thank her supervisors Professor A. W. Goldie and Dr. R. B. J. T. Allenby for their help and encouragement and the S.R.C. for the provision of a grant.

2. Preliminaries. We need the following group theoretic results. For any group $G$, we define $\Delta^{+}(G)$ by

$\triangle^{+}(G)=\{x \in G: x$ has only a finite number of conjugates in $G$ and $x$ has finite order $\}$.

Lemma 1. If $G=A *_{H} B$ then $\Delta^{+}(G) \leq \Delta^{+}(H)$.

Glasgow Math. J. 21 (1980) 135-138. 
Proof. This is straightforward.

TheOREM 1. Let $G=A *_{H} B$, where $H$ is a group with minimum condition. If $H$ is not normal in $G$, and if $H$ has no non-trivial subgroups which are normal in $G$, then there exists $g \in G$ such that $\mathrm{g}^{-1} \mathrm{Hg} \cap \mathrm{H}=1$.

Proof. See [3, proof of Theorem 1].

THEOREM 2. Let $P$ be a group having subgroups $A_{i}(i \in I)$ which intersect pairwise in a common subgroup $B$. That is, for $i, j \in I$ with $i \neq j$, we have $A_{i} \cap A_{i}=B$. If every element $p \in P$ has a normal form as defined in the introduction and if normal forms of different lengths represent different elements of $P$, then $P$ is the free product of the $A_{i}$ amalgamating $B$.

Proof. See [8, p. 511].

\section{The main result.}

Theorem 3. Let $R$ be a ring with 1 and let $G=A *_{H} B$, where $H$ is finite. If $G$ is not finitely generated and if $I$ is a non-zero ideal of $R(G)$, then there exist subgroups $C$ and $D$ of $G$, strictly containing the finite normal subgroup $\Delta^{+}(G)$, such that $I \cap R(M) \neq 0$, where $M=C *_{\triangle^{+}(G)} D$.

Proof. By Lemma $1, \triangle^{+}(G) \leq H$ and is hence a finite normal subgroup of $G$. Now $\triangle^{+}\left(G / \triangle^{+}(G)\right)=1$ (see $\left[9,19.3\right.$, p. 81]) and $G / \triangle^{+}(G)=A / \triangle^{+}(G) *_{\mathrm{H} / \Delta^{+}(G)} B / \triangle^{+}(G)$. Since $\triangle^{+}\left(G / \triangle^{+}(G)\right)=1$, no non-trivial subgroup of $H / \triangle^{+}(G)$ is normal in $G / \triangle^{+}(G)$. Hence we know from theorem 1 that there exists $\bar{g} \in G / \Delta^{+}(G)$ such that $\bar{g}^{-1}\left(H / \triangle^{+}(G)\right) \bar{g} \cap H / \triangle^{+}(G)=1$. Let $g$ be an inverse image of $\bar{g}$ in $G$. Then $g^{-1} H g \cap H \leq$ $\triangle^{+}(G)$. Since $\triangle^{+}(G)$ is normal in $G$ and a subgroup of $H, g^{-1} H g \cap H=\triangle^{+}(G)$. As $G$ is not finitely generated, either $A$ is not finitely generated or $B$ is not finitely generated. We suppose the former. If $g$ has $A$ - form, choose $b \in B, b \notin H$. Then if $h \in g^{-1} b^{-1} H b g \cap H$, $h=g^{-1} b^{-1} h_{1} b g$ for some $h_{1} \in H$. Since $g$ is $A-, b^{-1} h_{1} b \in H$ and so $h \in g^{-1} H g \cap H=$ $\triangle^{+}(G)$. Thus $g^{-1} b^{-1} H b g \cap H=\triangle^{+}(G)$ and we may assume that $g$ has $B$ - form. Similarly we may suppose without loss of generality that $g$ has $B B$ form, if $H$ is not normal in $A$, and that $g$ has $B A$ form otherwise. Let $0 \neq \theta \in I$ and let $L=\langle\operatorname{supp} \theta, H\rangle$. Since $A$ is not finitely generated and $L$ is finitely generated we can choose $a \in A$ such that for all $c \in L$, $a^{-1} c a$ has $A A$ form or $a^{-1} c a \in H$. Let $C=\mathrm{g}^{-1} a^{-1} L a g$. If $H$ is not normal in $A, g$ has $B B$ form and so for $c \in C, c$ has $B B$ form or $c \in \triangle^{+}(G)$. If $H$ is normal in $A$, either $H$ is not normal in $B$ or $H$ is normal in $G$. In the first case, the argument is analogous to what follows with elements of $C$ having $A A$ form or belonging to $\triangle^{+}(G)$. In the second case, $H=\triangle^{+}(G)$ and the result is trivial. Thus we may assume that $H$ is not normal in $A$. Hence we can choose $a_{1} \in A$ such that $a_{1} \notin H$ and $a_{1}^{2} \notin H$. Let $b \in B$ with $b \notin H$ and let $D=\left\langle a_{1} b a_{1}, \Delta^{+}(G)\right\rangle$. Elements of $D$ will have the form $d\left(a_{1} b a_{1}\right)^{n}$, where $d \in \Delta^{+}(G)$. Consider the group $M=\langle C, D\rangle$. Any element of $M$ can be written

$$
d\left(a_{1} b a_{1}\right)^{n_{1}} m_{1}\left(a_{1} b a_{1}\right)^{n_{2}} m_{2} \ldots m_{n},
$$


where $m_{i}$ has $B B$ form for $i=1, \ldots, n-1, n_{i}$ is an integer for $i=1, \ldots, n, n_{i} \neq 0$ for $i=2, \ldots, n$ and $m_{n}$ has $B B$ form or $m_{n}=1$. Thus every element of $M$ has a normal form and normal forms of different lengths represent different elements in $M$. Hence by Theorem 2, $M=C *_{\Delta^{+}(G)} D$. Since $\triangle^{+}(G)$ is normal in $G$ it is normal in $M$ and $0 \neq g^{-1} a^{-1} \theta a g \in R(M) \cap I$, giving the required result.

Note. It is not known to the author whether the condition in Theorem 3, that $G$ be not finitely generated, is necessary.

4. Applications. When $H$ is a normal subgroup of $G=A *_{H} B$ we have the following results for $J R(G)$.

Theorem 4. Let $R$ be a ring and let $G=A *_{H} B$ with $H$ normal in $G$ and $|A: H| \neq 2$ or $|B: H| \neq 2$. Suppose that $R(H)$ is a right (left) noetherian ring. Then $J R(G)=0$ if and only if $R(H)$ is semiprime.

Theorem 5. Let $K$ be a field of characteristic $p \neq 0$. Let $G=A *_{H} B$ with $H$ normal in G. Suppose that $H$ is a polycyclic-by-finite group. Then $J K(G)=N K(H) K(G)=N K(G)$.

(Note that if the characteristic of $K$ is 0 , then $J K(G)=N K(G)=0$ by Theorem 4 and $[9,3.3$, p. 9].)

These results can be obtained by modifying the proof of [4, Theorem 2], and considering the case $|A: H|=|B: H|=2$ separately. Details may be found in [5].

We use our main theorem to prove

TheORem 6. Let $G=A *_{H} B$, where $H$ is a finite group, and let $R$ be a right noetherian ring. If $G$ is not finitely generated then

(i) $R(G)$ is semiprimitive if and only if $R(G)$ is semiprime,

(ii) if $R$ is a field, $J R(G)=N R\left(\triangle^{+}(G)\right) R(G)$.

Proof. If $H$ is normal in $G$, the result follows from Theorem 4 and Theorem 5. Thus we may assume that $H$ is not normal in $G$. Let $0 \neq \theta \in J R(G)$; then, by the proof of Theorem 3, there is $g \in G$ and $a \in A$ with $g^{-1} a^{-1} \theta a g \in R(M) \cap J R(G)$, where $M=$ $C *_{\triangle^{+}(G)} D$. But $R(M) \cap J R(G) \subseteq J R(M)$ (see [9, 16.9, p. 68]). Thus $J R(M) \neq 0$. Since $\triangle^{+}(G)$ is finite, $R\left(\triangle^{+}(G)\right)$ is right noetherian and so Theorem 4 shows that $R\left(\triangle^{+}(G)\right)$ is not semiprime. Now $N R\left(\triangle^{+}(G)\right)$ is nilpotent and so $N R\left(\Delta^{+}(G)\right) R(G)$ is a nilpotent ideal in $R(G)$ and $R(G)$ is not semiprime. Clearly if $R(G)$ is not semiprime $R(G)$ is not semiprimitive and we have proved (i). For (ii) we apply Theorem 5 to obtain $J R(M)=$ $N R\left(\triangle^{+}(G)\right) R(G)=N R(G)$. Thus $g^{-1} a^{-1} \theta a g \in N R\left(\triangle^{+}(G)\right) R(G)$. Since $N R\left(\triangle^{+}(G)\right)$ is a nilpotent ideal of $R\left(\triangle^{+}(G)\right)$ and invariant under automorphisms, $N R\left(\triangle^{+}(G)\right) R(G)$ is a nilpotent ideal of $R(G)$. Thus $\theta \in N R\left(\triangle^{+}(G)\right) R(G)$ and we have shown that $J R(G) \subseteq$ $N R\left(\triangle^{+}(G)\right) R(G)$. $N R\left(\triangle^{+}(G)\right) R(G) \subseteq J R(G)$ since it is nilpotent, and we have the required equality.

The following result is a special case of Theorem 3.4 in [1]. 
Theorem 7. Let $K$ be a field and $G=A *_{H} B$, where $H$ is finite and normal in $G$. Then $Z K(G)=N K(G)$.

We use this to obtain

TheOREM 8. Let $K$ be a field and $G=A *_{H} B$ with $H$ finite and $G$ not finitely generated. Then $Z K(G)=N K(G)$.

Proof. If $H \triangleright G$, the result follows by Theorem 7. Thus we may assume that $H$ is not normal in $G$. Let $0 \neq \theta \in Z K(G)$. Then, by the proof of Theorem $3, \mathrm{~g}^{-1} a^{-1} \theta a g \in$ $K(M) \cap Z K(G)$, where $M=C *_{\triangle^{+}(G)} D$. Thus $g^{-1} a^{-1} \theta a g \in K(M)=Z K(M)=N K(M)$ by Theorem 7 and [2, Lemma 4.7]. Now since $\triangle^{+}(G)$ is finite and normal in $M, N K(M)=$ $N K\left(\triangle^{+}(G)\right) K(M)$, which is a nilpotent ideal invariant under automorphisms. Thus $\theta \in N K\left(\triangle^{+}(G)\right) K(M)$ and hence $\theta \in N K\left(\triangle^{+}(G)\right) K(G) \subseteq N K(G)$. Thus $Z K(G) \subseteq N K(G)$ and hence $Z K(G)=N K(G)$.

\section{REFERENCES}

1. K. A. Brown, The singular ideals of group rings, II, Quart. J. Math. Oxford Ser. 229, (1978), 187-197.

2. W. D. Burgess, Rings of quotients of group rings; Canad. J. Math, 21 (1969), 865-875.

3. D. Ž. Djoković and C. Y. Tang, On the Frattini subgroup of the generalized free product with amalgamation, Proc. Amer. Math. Soc. 32 (1972), 21-23.

4. C. R. Jordan, The Jacobson radical of the group ring of a generalised free product, $J$. London Math. Soc. (2) 11 (1975), 369-376.

5. C. R. Jordan, Ph.D. Thesis, University of Leeds, 1975.

6. A. I. Lichtman, Ideals in group rings of free products with amalgamations and of HNN extensions, preprint.

7. W. Magnus, A. Karras and D. Solitar, Combinatorial group theory (Interscience, 1966).

8. B. H. Neumann, An essay on free products of groups with amalgamations, Philos. Trans. Roy. Soc. London Ser. A 246 (1954), 503-554.

9. D. S. Passman, Infinite group rings (Marcel Dekker, 1971).

C/O DR. D. A. JoRDAN

Department of Pure Mathematics

The University of ShefField

THE HiCKS BUILding

SHEFFIELD S3 7RH 